\title{
IFT80 is required for stem cell proliferation, differentiation, and odontoblast polarization during tooth development
}

Xue Yuan ${ }^{1}$, Xu Cao ${ }^{2}$ and Shuying Yang ${ }^{1,3}$

\begin{abstract}
Primary cilia and intraflagellar transport (IFT) proteins control a wide variety of processes during tissue development and homeostasis. However, their role in regulation of stem cell properties during tooth development remains elusive. Here, we revealed that dental pulp stem cells (DPSCs) express IFT80, which is required for maintaining DPSC properties. Mice with deletion of IFT80 in odontoblast lineage show impaired molar root development and delayed incisor eruption through reduced DPSC proliferation and differentiation, and disrupted odontoblast polarization. Impaired odontoblast differentiation resulted from disrupted hedgehog $(\mathrm{Hh})$ signaling pathways. Decreased DPSC proliferation is associated with impaired fibroblast growth factor 2 (FGF2) signaling caused by loss of IFT80, leading to the disruption of FGF2-FGFR1-PI3K-AKT signaling in IFT80-deficient DPSCs. The results provide the first evidence that IFT80 controls tooth development through influencing cell proliferation, differentiation, and polarization, and Hh and FGF/ AKT signaling pathways, demonstrating that IFT proteins are likely to be the new therapeutic targets for tooth and other tissue repair and regeneration.
\end{abstract}

\section{Introduction}

Tooth development, like many other organs' formation, requires multi-lineage cells controlled by different signaling pathways. During tooth formation, a subpopulation of mesenchymal cells differentiates into odontoblasts, which are the columnar polarized cells located at the outer edges of dental pulp, expressing dentin matrix protein 1 (DMP1) and dentin sialophosphoprotein (DSPP), and producing dentin. Ameloblasts are derived from dental epithelium and secrete enamel matrix, which is the hardest and highly mineralized tissue. Dental pulp is the soft tissue inside the tooth, and their function is to support dentin formation and regeneration ${ }^{1,2}$.

\footnotetext{
Correspondence: Shuying Yang (shuyingy@upenn.edu)

${ }^{1}$ Department of Oral Biology, School of Dental Medicine University of Buffalo, State University of New York, Buffalo, NY, USA

${ }^{2}$ Department of Orthopedic Surgery, Johns Hopkins University School of Medicine, Baltimore, MD, USA

Full list of author information is available at the end of the article.

Edited by $Y$. Wang
}

Primary cilia are highly conserved microtubule-based solitary organelles that are present on almost all vertebrate cells. Formation and function of primary cilia require intraflagellar transport (IFT) proteins and other ciliary proteins ${ }^{3,4}$. Mutation of those proteins usually causes cilia defects, leading to a wide range of diseases called ciliopathies. These disorders target multiple tissues, of which bone and tooth are most common tissues ${ }^{5-7}$. The arising evidence demonstrates that primary cilia play important roles in tooth development ${ }^{8,9}$. For example, Wnt1-Cre; Kif3a ${ }^{f l f l}$ mice (deletion of ciliary motor-Kif3a in dental mesenchyme) show impaired incisor and molar development ${ }^{9,10}$. Abnormal molar development was also found in $E v c^{-1-11}$ and IFT88 ${ }^{\text {orpk }}$ mice $^{7}$. Hypomorphic alleles of IFT88 $\left(\mathrm{Tg} 737^{\text {orpk }}\right)$ showed increased Hh signaling and formation of supernumerary molars in the diastema of both the lower and upper jaws ${ }^{12,13}$. Previously, we reported that IFT80, one of the IFT complex B proteins, promotes osteogenesis ${ }^{14}$ and deletion of IFT80 in osteoblast precursor cells leads to decreased bone mass

\section{(c) The Author(s) 2019}

(c) (i) Open Access This article is licensed under a Creative Commons Attribution 4.0 International License, which permits use, sharing, adaptation, distribution and reproduction c. in any medium or format, as long as you give appropriate credit to the original author(s) and the source, provide a link to the Creative Commons license, and indicate if changes were made. The images or other third party material in this article are included in the article's Creative Commons license, unless indicated otherwise in a credit line to the material. If material is not included in the article's Creative Commons license and your intended use is not permitted by statutory regulation or exceeds the permitted use, you will need to obtain permission directly from the copyright holder. To view a copy of this license, visit http://creativecommons.org/licenses/by/4.0/. 


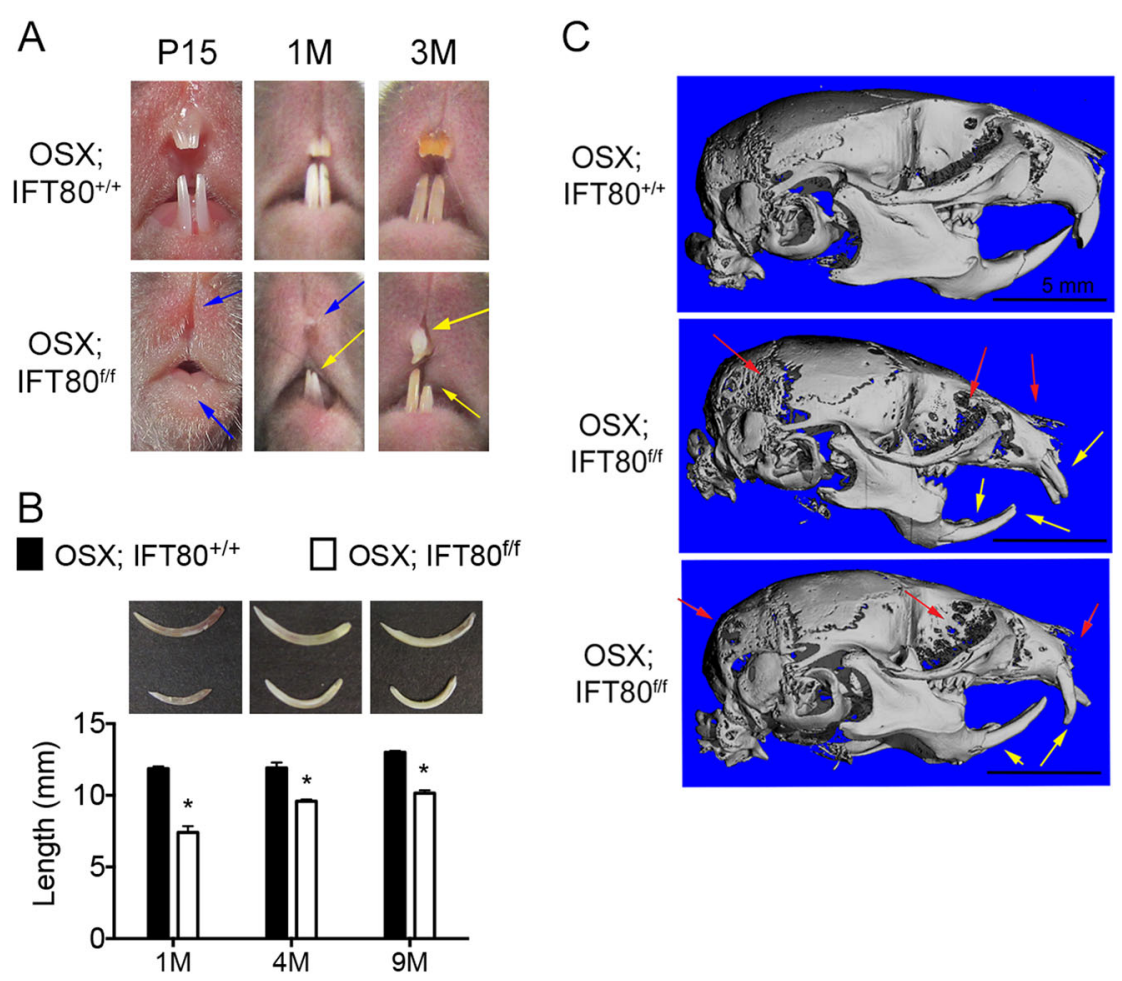

Fig. 1 OSX;IFT80 ${ }^{f / f}$ mice show impaired incisor eruption and development. a Photographic analysis of incisor development. Blue arrows indicate missing incisors. Yellow arrows indicate abnormal incisors. b Average length of lower incisors $\left(n=6,{ }^{*} p<0.001\right.$ versus OSX,IFT $80^{+/+}$at different time points). c Side view of micro-CT to show the malocclusion (yellow arrows) and impaired craniofacial mineralization in $1 \mathrm{M}$ OSX;IFT8 ${ }^{f / f}$ mice (red arrows). Scale bars represent $5 \mathrm{~mm}$. Data are expressed as mean $\pm \mathrm{SEM} ;{ }^{*} p<0.001$

with impaired osteoblast differentiation through regulating Hh signaling pathway ${ }^{15}$. However, their role in regulation of stem cell properties and odontogenesis during tooth development remains elusive.

Fibroblast growth factors (FGFs) are growth factors that are important for bone and tooth development and regeneration ${ }^{16}$. There are 22 FGF proteins that interact with four highly conserved transmembrane tyrosine kinase receptors (FGFR1-4). Among FGFs, FGF2 is highly expressed in odontoblast lineage cells ${ }^{17}$. Several studies supported that FGF2 promotes the pulp cell proliferation, self-renewal, migration, and early differentiation ${ }^{18-22}$. However, the relationship between FGF signaling and primary cilia/IFT proteins has not been established.

In this study, we generated IFT80 conditional knockout mouse by breeding IFT80-floxed mice with Osterix (OSX)Cre transgenic mice and examined their phenotypic and molecular changes in tooth development. In addition, we determined the mechanism by which IFT80 regulates dental stem cell proliferation, differentiation, and polarization during tooth development. We found for the first time that IFT80 governs tooth development through influencing DPSC proliferation, differentiation, and odontoblast polarization by regulating $\mathrm{Hh}$ and FGF/AKT signaling pathways, demonstrating that IFT proteins are likely new therapeutic targets for tooth and other tissue repair and regeneration.

\section{Results \\ Conditional deletion of IFT80 impaired incisor development}

OSX is a transcription factor during osteoblast differentiation from stem cells and OSX+ cells are essential for bone development ${ }^{23}$. Recent studies demonstrate that OSX is also expressed in pulp cells during differentiation of odontoblasts ${ }^{24,25}$. Therefore, we generated OSX;IFT8 $0^{f / f}$ mice to study the function of IFT80 in tooth development. We observed that incisors were completely absent in 15day-old OSX;IFT $80^{f / f}$ mice, and severely underdeveloped and malocclusioned in 1-month-old and 3-month-old OSX;IFT $80^{f / f}$ mice (Fig. 1a). The average incisor eruption age was around postnatal day 7 in OSX;IFT80 ${ }^{+/+}$mice, whereas it was delayed to postnatal day 14 for lower incisors and postnatal day 21 for upper incisors in OSX; $I F T 80^{f / f}$ mice. Mandibular incisors were isolated from their sockets for morphological analysis. OSX;IFT8 8 f/f incisors were obviously shorter but more curved at all examined time points (Fig. 1b). The mean length of lower 

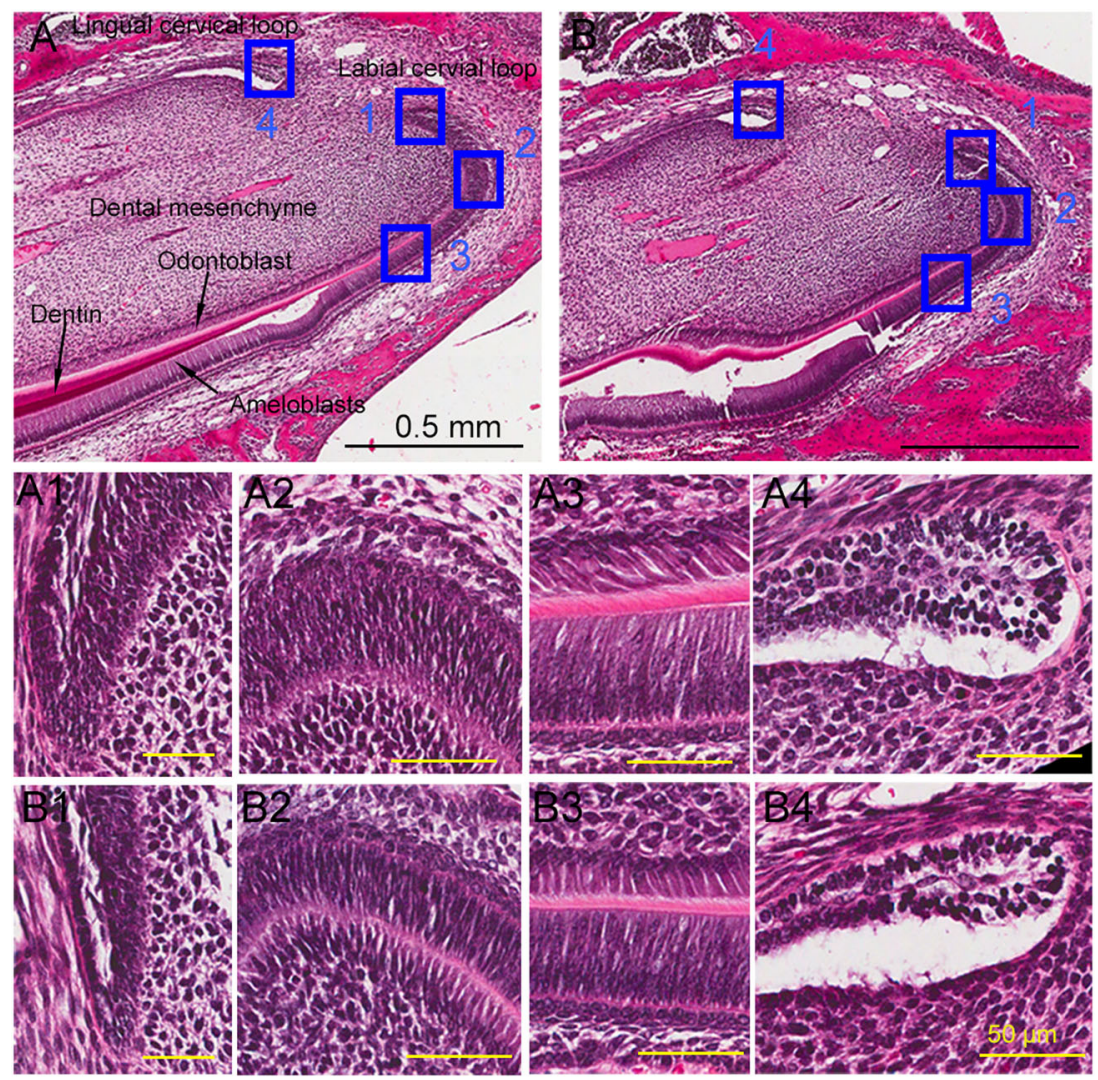

C
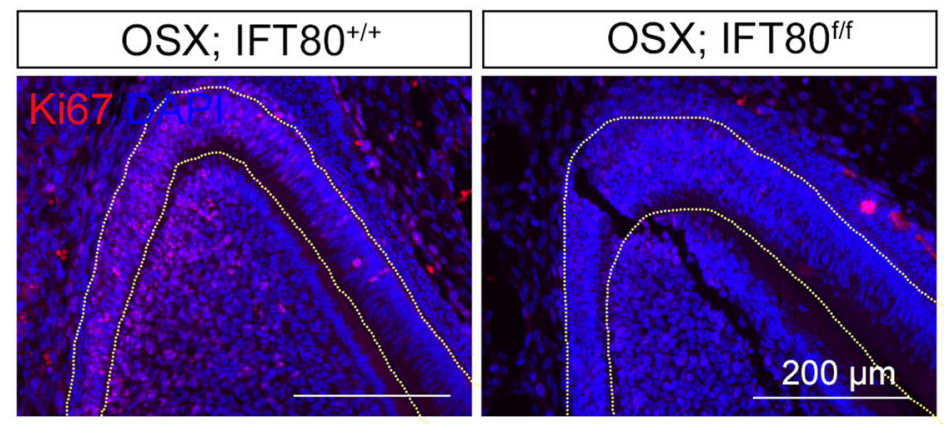

Fig. 2 Pulp cell proliferation in the cervical loop is impaired in OSX;IFT80 $0^{\mathbf{f f f}}$ mice. $\mathbf{a}$, b Hematoxylin and eosin staining of the proximal incisor

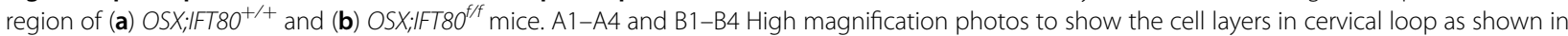
$\mathbf{a}$ and $\mathbf{b}$ (blue boxes). Scale bars represent $0.5 \mathrm{~mm}$ (black) or $50 \mu \mathrm{m}$ (yellow). c Ki67 (red) staining of cervical loop section of OSX;IFT80 ${ }^{+/+}$and OSX; IFT80 ${ }^{f / f}$ mice. DAPI staining is used as a counterstain. Scale bars represent $200 \mu \mathrm{m}$

incisors in OSX;IFT80 $0^{f / f}$ mice was only 0.61 -fold of that in OSX;IFT80 ${ }^{+/+}$mice at 1 month old (Fig. 1b). Examination of skulls by micro computed tomography showed the malocclusion and defects in both mandibular and maxillary incisors in OSX;IFT8 ${ }^{f / f}$ mice (Fig. 1c). These data suggest that IFT80 is critical for incisor development. Notably, OSX;IFT80 fff mice also showed markedly decreased bone mass in craniofacial bones as well as alveolar bones (Fig. 1c).

\section{Conditional deletion of IFT80 impaired pulp cell proliferation in the cervical loop}

Histological analysis revealed that the cervical loops and odontoblast layers were smaller in OSX;IFT80 ${ }^{f / f}$ mice compared with those in OSX;IFT80 $0^{+/+}$mice (Fig. 2a, $\mathrm{A} 1-\mathrm{A} 4$ and $2 \mathrm{~b}, \mathrm{~B} 1-\mathrm{B} 4)$, suggesting that the proliferation might be compromised in this area. Therefore, we performed Ki67 staining to detect cell proliferation. As we expected, the results showed that proliferating cells were 
A

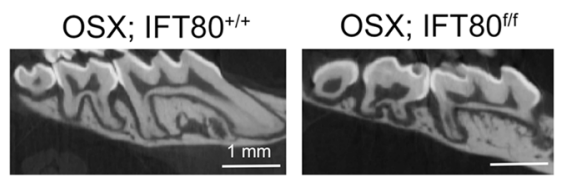

$\mathrm{B}$

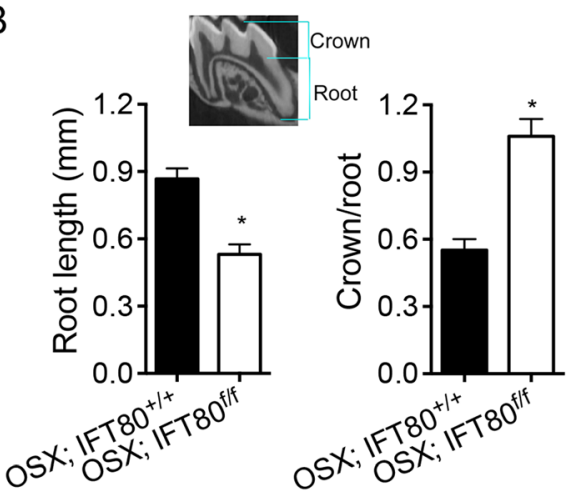

C osX; IFT80+/+
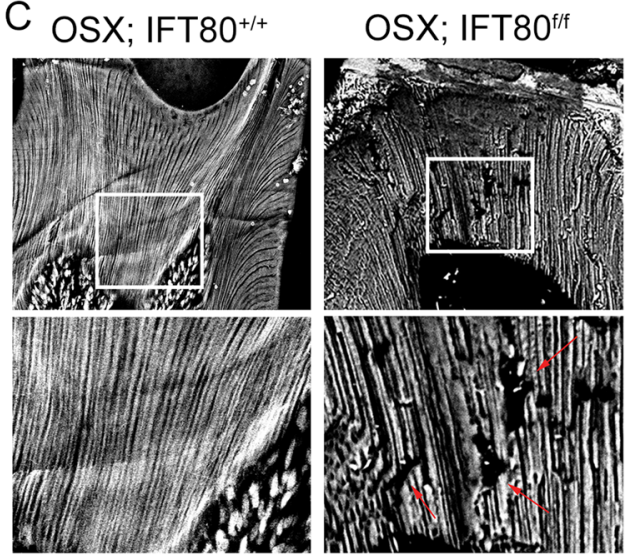

Fig. 3 OSX;IFT8 ${ }^{f / f}$ mice show shorter molar root, less mineralized dentin, and disrupted odontoblast differentiation. a Micro-tomographic view of the molar root. Scale bars represent $1 \mathrm{~mm}$. b Measured root length and calculated crown/root ratio $(n=3)$. c Backscattered electron analysis of dentin tubules and mineralization. Red arrows indicate defect of dentinal tubules. Lower panels are enlarged images of the boxed areas in upper panels. $\mathbf{d}$ Hematoxylin and eosin staining of the molar root. D dentin, OD odontoblasts, P pulp, N nucleus. In OSX;IFT $80^{+/+}$mice, the odontoblasts were highly polarized and attached to each other by their terminal webs (cyan arrow). Lower panels are enlarged images of upper panels. Scale bars represent $60 \mu \mathrm{m}$ (upper) or $30 \mu \mathrm{m}$ (lower). e Dentin matrix protein 1 (DMP1) and dentin sialophosphoprotein (DSPP) expression in the dental pulp. The expression of DMP1 and DSPP was normalized to GAPDH expression $(n=3)$. Data are expressed as mean \pm SEM; ${ }^{*} p<0.0001$

significantly reduced in the cervical loop and the dental pulp in OSX;IFT80 f/f mice compared to OSX;IFT80 control mice (Fig. 2c). Together, these data implied that IFT80 is required for the odontoblast lineage cell proliferation and incisor growth.

\section{Conditional deletion of IFT80 caused shorter molar root, less mineralized dentin, and disrupted odontoblast differentiation}

We next examined molar development and found that molars were normally erupted in both OSX;IFT80 fff and OSX;IFT80 $0^{+/+}$mice. The crowns of molars were well formed but the roots were shorter in OSX;IFT80 f/f mice compared with those in OSX;IFT80 $0^{+/+}$mice (Fig. 3a and Fig. S1A and S1B). Quantitative analysis of the root and crown length of first molars in mandible showed that the roots from OSX;IFT8 $0^{f / f}$ mice were significantly shorter than those from OSX;IFT80 ${ }^{+/+}$mice (Fig. 3b), whereas crown length was similar in both groups. Thus, the crown-to-root ratio was significantly increased in OSX; IFT8 $0^{f / f}$ mice (Fig. 3b).

To further uncover the role of IFT80 in dentin formation and mineralization, we performed backscattered electron analysis. OSX;IFT80 $0^{+/+}$mice had well-organized 
and patterned dentinal tubules. In contrast, the dentinal tubules in OSX;IFT8 $0^{f / f}$ mice were disorganized with less mineralization (Fig. 3c). Odontoblasts are responsible for the production of calcified dentin matrix; therefore, these data suggested that IFT80 is involved in odontoblast differentiation and function. We further observed odontoblast morphology in the early stage of root formation. At postnatal day 14, odontoblasts were elongated and highly polarized in the proximal portion of the root in OSX; IFT80 $0^{+/+}$mice (Fig. 3d), and the nuclei were polarized in the pulp side of the odontoblasts. These cells were attached to each other with their terminal webs (Fig. 3d, cyan arrows). However, in OSX;IFT8 $80^{f / f}$ mice, odontoblasts were disorganized along the proximal portion of the root. Some of the odontoblasts displayed a reversed cell orientation with the nuclei close to the dentin side of the odontoblasts (Fig. 3d). In addition to the morphology changes, we also found that the expression of odontoblast marker genes (DMP1 and DSPP) in dental pulp was strikingly reduced in $O S X ; I F T 80^{f / f}$ mice by quantitative PCR (qPCR) (Fig. 3e), confirming that IFT80 deletion impaired odontoblast differentiation.

\section{Deletion of IFT80 disrupted cilia formation in odontoblasts and dental pulp cells}

To gain insight into the mechanism that IFT80 deficiency caused defective dental pulp cell differentiation and tooth development, we first examined cilia formation (Fig. 4a). In molar section, cilia were found on almost every ameloblast and the cilia length was similar between OSX;IFT80 $0^{+/+}$mice and OSX;IFT8 $0^{f / f}$ mice (Fig. 4a, b, c) However, in OSX;IFT8 $0^{f / f}$ odontoblasts, cilia formation was severely disrupted. The ciliated cell population reduced to $20 \%$ in OSX;IFT $80^{f / f}$ mice compared with $80 \%$ in OSX;IFT8O ${ }^{+/+}$mice (Fig. 4b). Moreover, the cilia length of ciliated odontoblasts was significantly shorter in OSX; IFT80 $0^{f / f}$ mice than that in OSX;IFT80 ${ }^{+/+}$mice (Fig. 4c). Additionally, the ciliated cell population but not cilia length were also reduced in dental pulp cells in OSX; IFT $80^{f / f}$ mice (Fig. 4b, c).

\section{Deletion of IFT80 disrupted cilia formation and $\mathrm{Hh}$ signaling in DPSCs}

To further examine the role of IFT80 in dental pulp cell proliferation and differentiation in vitro, we isolated primary DPSCs from the dental pulp of mouse incisor. DPSCs isolated from $I F T 80^{f / f}$ mice were infected with adenovirus-expressing Cre recombinase to delete IFT80 (named as $I F T 80^{d / d}$ ). Adenovirus expressing green fluorescent protein (GFP)-infected $I F T 80^{f / f}$ DPSCs were used as control (still marked as IFT80 $0^{f / 5}$ ). Western blot and qPCR confirmed that Cre adenovirus transduction significantly reduced IFT80 expression in DPSCs (Figs. 5a, b).
We compared cilia formation between IFT8 ${ }^{f / f}$ and IFT8 $^{d / d}$ DPSCs by immunostaining. Consistent to the results from in vivo tooth slide staining (Fig. $5 \mathrm{a}, \mathrm{b}$ ), the results showed that the ciliated population in $I F T 80^{d / d}$ DPSCs was reduced to $<30 \%$ and the average cilia length was decreased to $1.56 \mu \mathrm{m}$ compared with $80 \%$ and $3.72 \mu \mathrm{m}$ in IFT80 $0^{f / f}$ DPSCs, respectively (Fig. 5c, d). These results demonstrated that IFT80 is required for cilia formation in both dental pulp cells and odontoblasts. Primary cilia are essential for Hh signaling transduction, and our previous study has demonstrated that IFT80 is required for Hh signaling transduction in osteoblast differentiation ${ }^{15}$; therefore, we tested Hh signaling activation in IFT8 $0^{f f f}$ and IFT80 ${ }^{d / d}$ DPSCs. As expected, sonic Hh (Shh) activated Gli promoter activity as well as Gli1 and Ptch1 expression in IFT80 $0^{f / f}$ DPSCs (Fig. 5e, f), indicating Hh pathway activation. However, these responses were impaired in IFT80 d/d DPSCs (Fig. 5e, f), confirming disrupted Hh signaling transduction in IFT80 ${ }^{d / d}$ DPSCs.

\section{Deletion of IFT80 impaired DPSC proliferation through defect of FGF signaling rather than Hh signaling}

Since our in vivo results suggested that OSX;IFT80 $0^{f / f}$ mice has less proliferating cells in dental pulp (Fig. 2c), we then compared the proliferation rate between IFT80 $0^{f / f}$ and IFT80 ${ }^{d / d}$ DPSCs by MTS assay and Ki67 staining. The results showed that IFT80 deletion inhibits DPSC proliferation (Fig. 6a, b). To further test if disrupted Hh signaling in IFT80 d/d DPSCs contributes to the DPSC proliferative defect, $\mathrm{Hh}$ agonist purmorphomine was used to stimulate DPCSs in both IFT80 f/f and IFT80 $0^{d / d}$ groups. The results showed that activation of Hh signaling by purmorphomine did not affect DPSC proliferation in both groups (Fig. 6c, d), suggesting that the pathway(s) other than Hh signaling existed to regulate DPSC proliferation and were affected by loss of IFT80.

FGF signaling regulates the proliferation of many types of cells including dental pulp cells ${ }^{18,26}$. We found that FGF2 dramatically promotes proliferation of IFT80 f/f DPSCs, but failed to increase the proliferation of $I F T 80^{d / d}$ DPSCs (Fig. 6e, f), indicating that deletion of IFT80 impaired FGF2 signaling transduction, which caused defective DPSC proliferation.

\section{Deletion of IFT80 inhibited DPSC proliferation via down- regulation of FGF-PI3K-AKT signaling}

Phosphorylated FGFR activates phosphatidylinositol 3-kinase (PI3K) and AKT pathway, which converts FGF signaling to the proliferative effect. Consistently, we found that, in response to FGF2 stimulation, AKT phosphorylation increased in $I F T 80^{f / f}$ DPSCs, which was inhibited by PD173074 (FGFR inhibitor) or API-2 (AKT inhibitor) treatment (Fig. 7a, b). In IFT80 ${ }^{d / d}$ DPSCs, AKT expression was comparable to IFT80 fff; however, AKT 
A
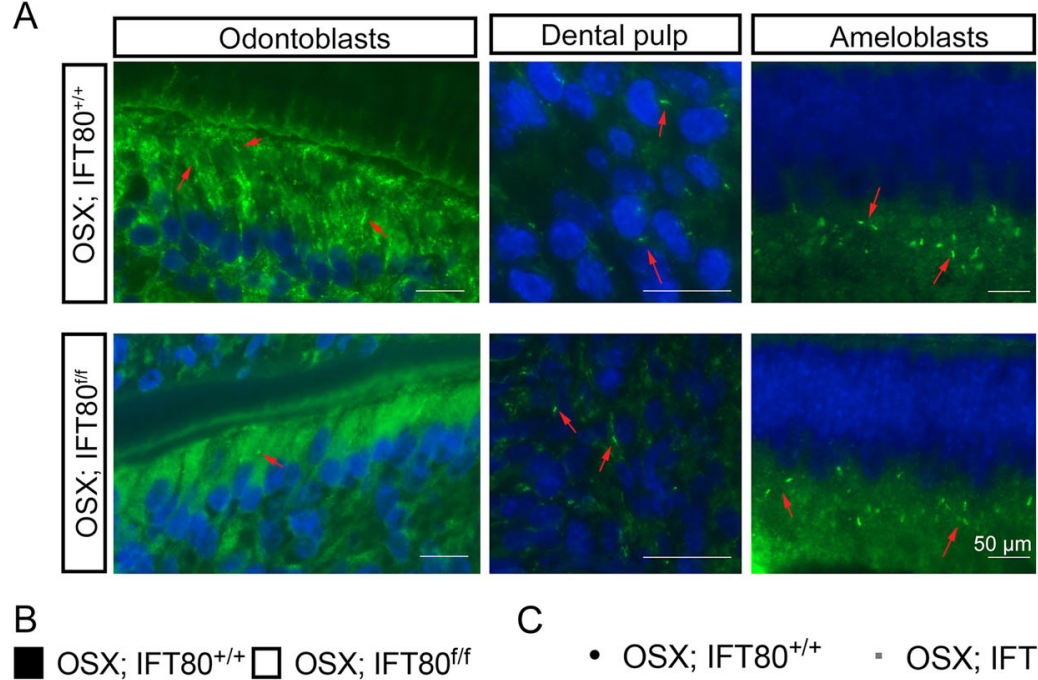

C
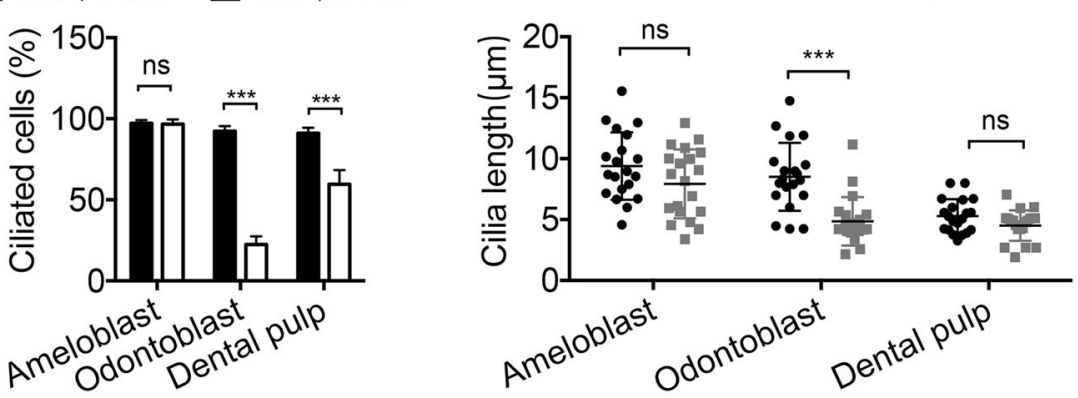

Fig. 4 Deletion of IFT80 disrupts cilia formation and Hh signaling transduction. a Immunofluorescence analysis of primary cilia in molar section (red arrow). Primary cilia were stained with acetylated a-tubulin (green) antibody. DAPI is used as a counterstain. Scale bars represent $50 \mu m$. b Calculated cilia percentage ( $n=3$ with at least 100 cells analyzed) is shown in a. c Calculated cilia length ( $n=20$ cells). Data are expressed as mean \pm SEM; ns, not statistically significant; ${ }^{* * *} p<0.0001$

phosphorylation level induced by FGF2 in $I F T 80^{d / d}$ DPSCs was much lower than that of IFT8 $0^{f / f}$ DPSCs (Fig. 7a, b), confirming that FGF2-FGFR signaling was blocked in IFT80 $0^{d / d}$ DPSCs. We further found that PD173074, LY294002 (PI3K inhibitor), API-2, or rapamycin (mammalian target of rapamycin (mTOR) inhibitor) inhibited the proliferation of FGF2-induced IFT80 $0^{f / f}$ DPSC but not IFT80 ${ }^{d / d}$ DPSCs (Fig. 7c). These data implied that IFT80 is essential for DPSC proliferation via regulating FGF2-FGFR1-PI3K-AKT-mTOR signaling pathway.

\section{Discussion}

Although primary cilia have been observed in DPSCs and odontoblasts more than a decade ago $^{27}$, the function of primary cilia and IFT proteins in DPSCs has not been well addressed. Our study reveals for the first time that IFT80 is required for tooth development by regulating DPSC proliferation, differentiation, and odontoblast polarization. Deletion of IFT80 in DPSCs impairs FGF2-FGFR-PI3K-AKT signaling that inhibits DPSC proliferation. Moreover, IFT80 deletion impairs cilia formation and Hh signaling transduction, which eventually inhibits DPSC differentiation and tooth development.

Primary cilium projects from the cell surface of most mammalian cells and coordinates of various signaling pathways during development and homeostasis. Although the exact roles of primary cilia in tooth development remain elusive, the emerging evidence indicates that primary cilia in both dental epithelium and mesenchyme are pivotal for early odontogenesis and later tooth development $^{28,29}$. A most recent study showed that IFT140, one of the IFT protein that formed IFT complex A, is highly expressed in odontoblasts and knockout of IFT40 leads to delayed and defective dentin formation due to poor odontogenic differentiation, abnormal primary cilia, and decreased Hh signaling ${ }^{30}$. In this study, we used an IFT80 conditional knockout mouse model, which is one of the components of IFT complex B, and found the similar dental phenotypes-reduced odontoblast proliferation and shorter root formation. Deletion of IFT140 or IFT80 all causes defects in cilia formation, suggesting that primary cilia, instead of individual IFT protein, play an essential role in the odontoblast proliferation and 


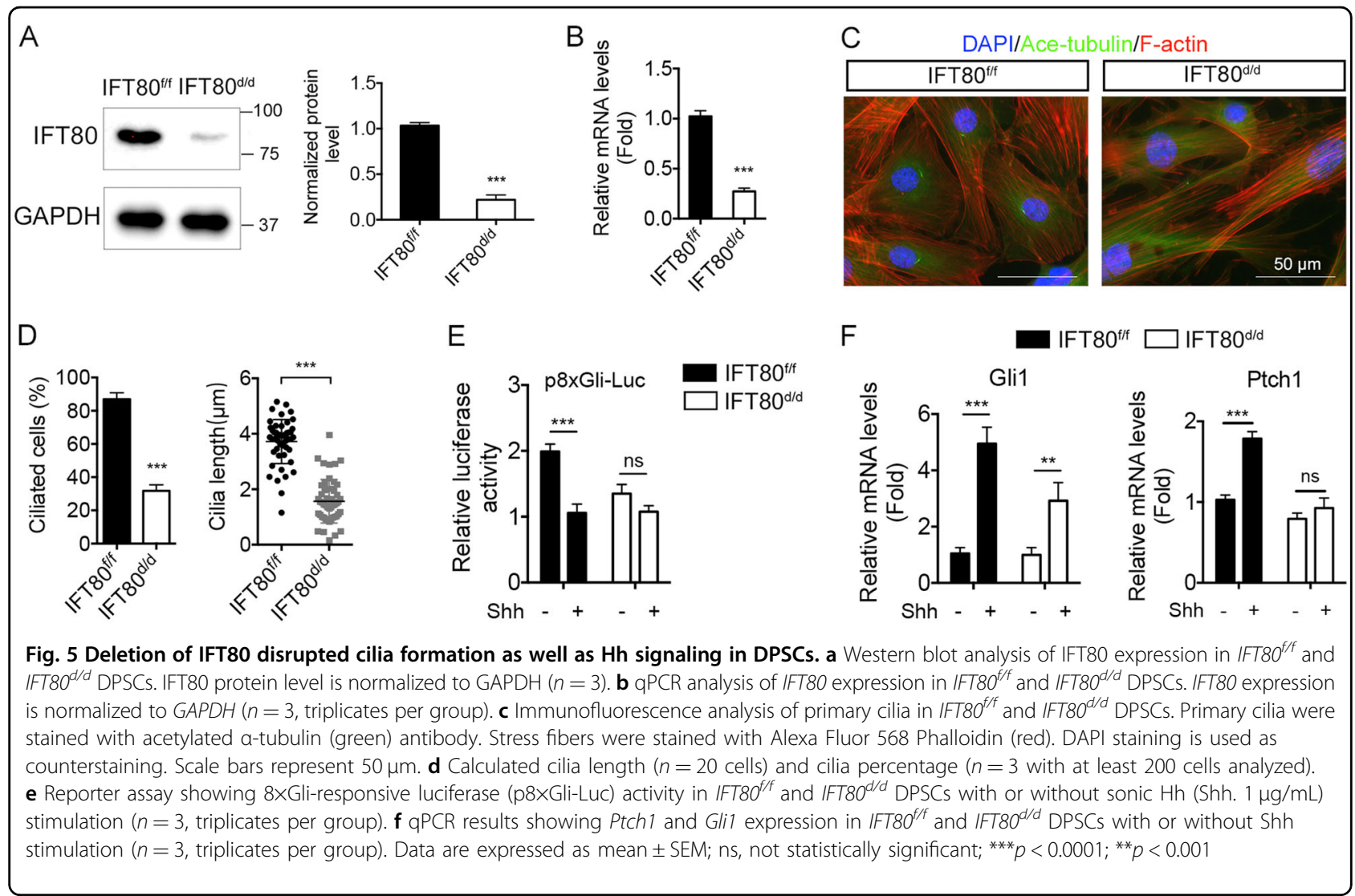

differentiation. However, inconsistently, hypomorphic alleles of IFT88 ( $\mathrm{Tg} 737^{\text {orpk }}$ ) showed increased Hh signaling and formation of supernumerary molars in the diastema of both the lower and upper jaws, indicating that IFT protein likely also regulates cilia-independent pathway during tooth development.

Hisamoto et al. ${ }^{31}$ studied the pattern of cilia in tooth germ and oral cavity and found that the cilia in odontoblasts first appears near the root apex and then extends along the odontoblast layer ${ }^{31}$. This might explain why we observed severe root formation defects in IFT80 knockout mice. Their study also indicated the stage-specific and region-specific cilia formation and cilia length regulation. A reasonable assumption is that the cell differentiation status determines the cilia length, which closely related to their sensory function ${ }^{29,31}$. In IFT80 knockout mice, we also observed the cilia length change in both pulp cells and odontoblasts (Fig. 4). Whether cilia length is directly associated with differentiation status needs further study. Our data here suggest that in addition to odontoblast differentiation and pulp cell proliferation (Fig. 2), odontoblast polarization (Fig. 3) requires functional cilia.

Primary cilium serves as a signaling hub and a variety of receptors and signaling proteins locate in cilia $^{3,32}$. For example, the key components of Hh signaling including
Patched1 (Ptch1), Smoothened, and Gli transcription factors are present in primary cilia ${ }^{33,34}$. It is well known that $\mathrm{Hh}$ signaling transduction requires IFT proteins and other ciliary protein ${ }^{35}$. In our previous study, we reported that IFT80 is essential for Hh signaling in osteoblasts, chondrocytes, and bone marrow derived mesenchymal stem cells ${ }^{14,15,36,37}$. Consistently, in this study, we found that IFT80 deletion in DPSCs disrupts cilia formation and Hh signaling transduction (Figs. 4 and 5). In addition to Hh signaling, emerging studies have suggested that IFT proteins and primary cilia mediate platelet-derived growth factor signaling ${ }^{38}$, Notch $^{39}$, insulin-like growth factor $^{40,41}$, and epidermal growth factor ${ }^{32}$ signaling pathways. Besides these critical pathways, cilia formation is also regulated by other signaling pathways like FGF signaling. Mutation of FGFR1, a receptor of FGF signaling, in zebrafish shortens cilia ${ }^{42}$. Whether IFT proteins regulate FGF signaling was unknown. In our study, we found that loss of IFT80 in DPSCs dramatically blocked FGF2induced DPSC proliferation (Fig. 6), indicating that IFT80-mediated primary cilia formation is important for FGF2 transduction. It is still not clear how primary cilia loss effects FGF2 signaling. One possible explanation is that the receptor for FGF2 is normally located in primary cilia and loss of cilia leads to the dysregulation of FGFR. 


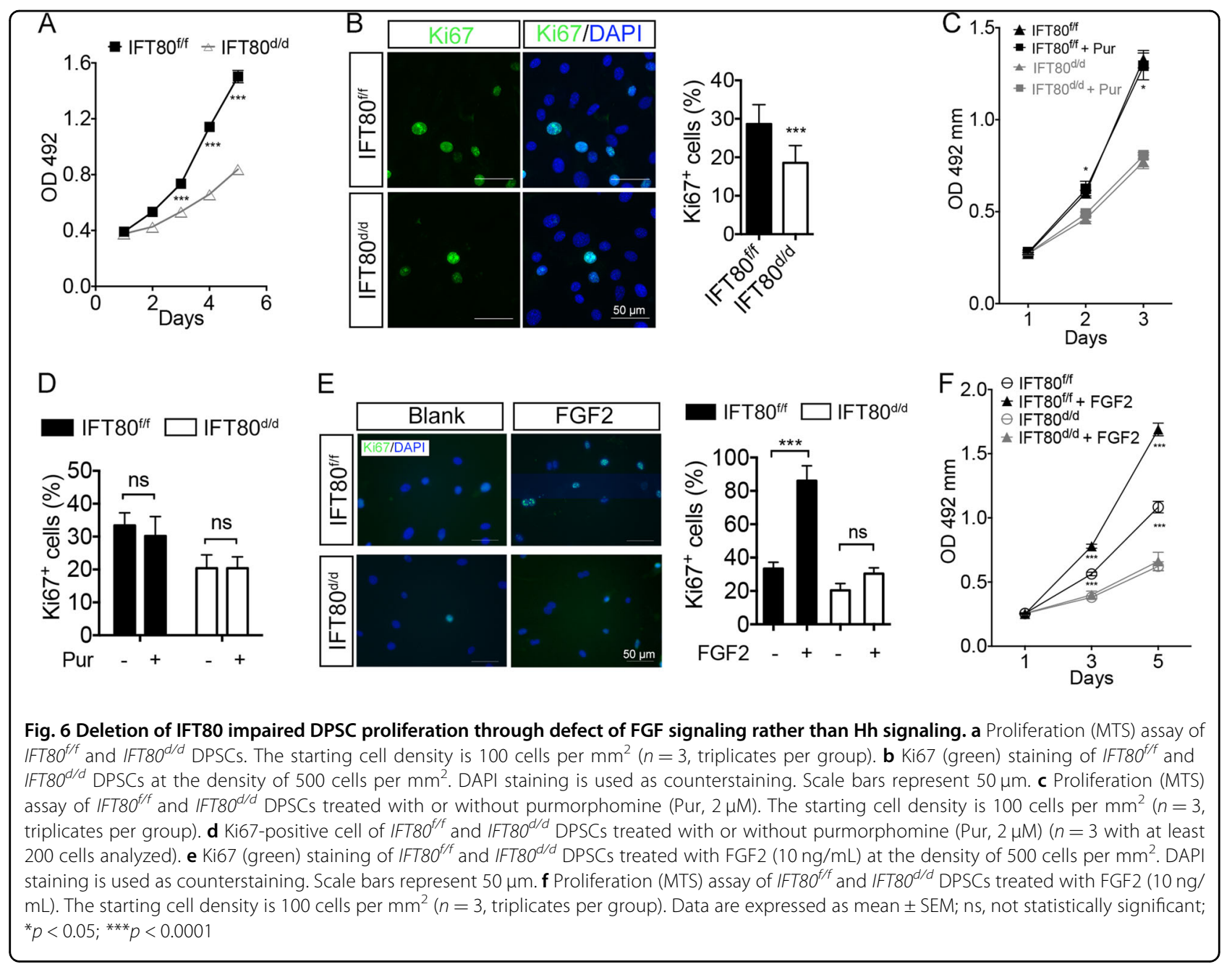

Another possible explanation is that primary cilia indirectly effect FGF2 signaling by targeting the downstream signaling, such as PI3K-AKT.

FGF2 signaling has been recognized as an important signaling that regulate both cell proliferation and cell differentiation; however, its functions are complicated and stage-dependent ${ }^{18,22,43}$. Our work showed that IFT protein/primary cilia regulates the FGF2 signaling, which indicated a new regulatory mechanism of FGF2 signaling during odontogenesis and tooth development. Further study regarding how IFT and cilia regulate stem cell selfrenewal and differentiation is going on. Collectively, this study demonstrated for the first time that IFT80 is a critical regulator of DPSC proliferation and tooth development. IFT80 maintains cilia formation and stimulates DPSC proliferation. Thus, we revealed a novel role and mechanism of IFT 80 in the regulation of DPSC and tooth development, and provided new insights for bone and tooth regenerative therapeutic design and therapy.

\section{Materials and methods Mice}

All experiments performed on mice were approved by the University at Buffalo Institutional Animal Care and Use Committee. The generation of IFT8 fff mice model (two LoxP sites flanking exon 6 of IFT80) was previously described $^{15}$. OSX-Cre transgenic mice (also known as OSX1-GFP::Cre (Jackson \#006361)) were crossed with IFT $80^{f / f}$ mice to generate OSX-Cre;IFT8 ${ }^{f /+}$ mice, which

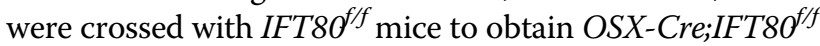
mice (named as OSX;IFT8 $0^{f / f}$ ). OSX-Cre mice were used as experimental controls (named as OSX;IFT80 ${ }^{+/+}$).

\section{Reagents}

Recombinant mouse Shh N-terminus $(1 \mu \mathrm{g} / \mathrm{mL}, \mathrm{R} \& D$ Systems, Minneapolis, MN, USA) or purmorphomine $(2 \mu \mathrm{M}$, Tocris Bioscience, 4551) was used to activate $\mathrm{Hh}$ signaling. FGF2 $(10 \mathrm{ng} / \mathrm{mL}$, R\&D Systems, Minneapolis, MN, USA) was used to activate FGF signaling. PD173074 $(1 \mu \mathrm{M}$, Tocris, 3044) was used to inhibit FGFR. LY294002 


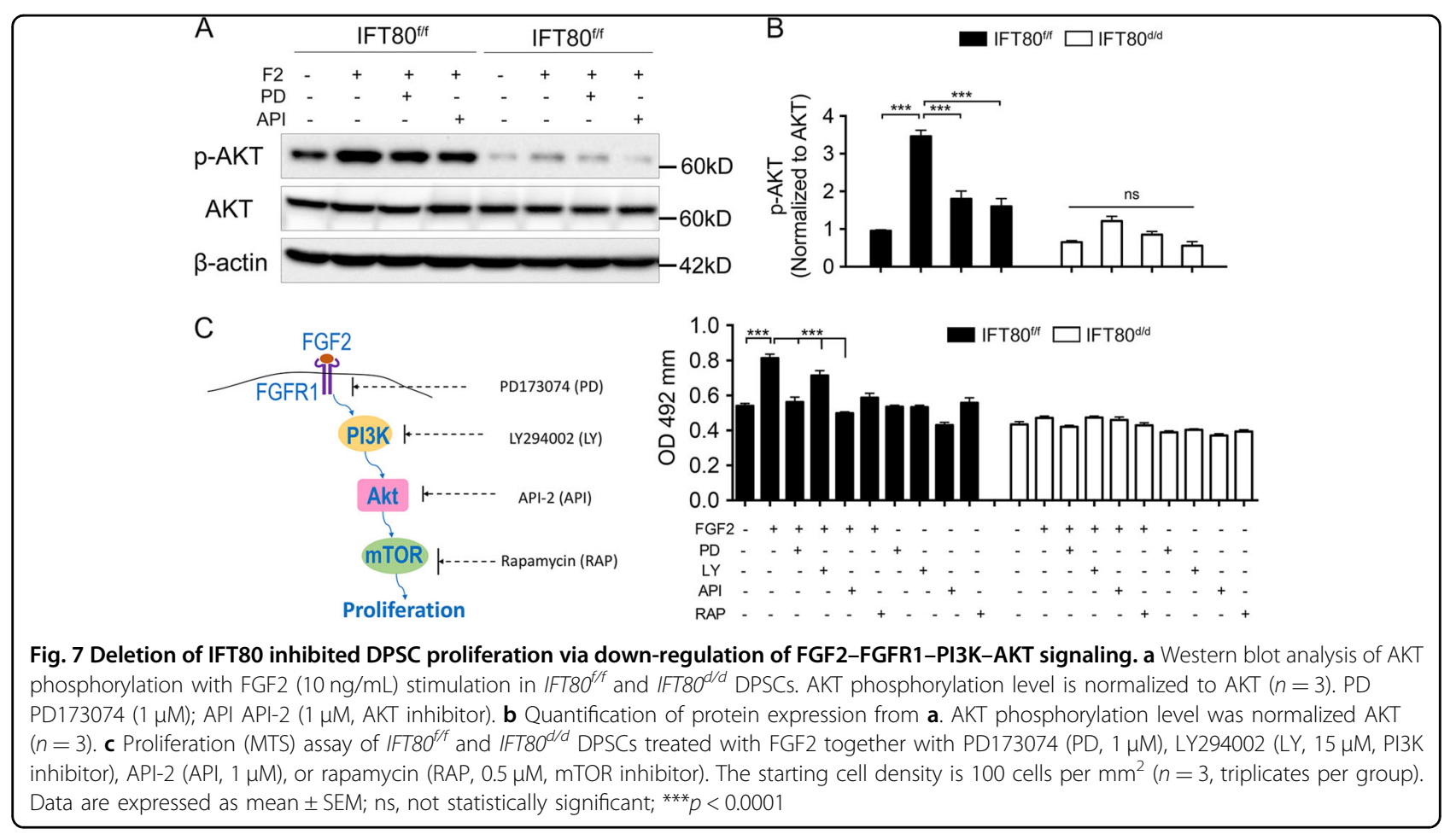

(15 $\mu \mathrm{M}$, Sigma, L9908), API-2 (1 $\mu \mathrm{M}$, Tocris, 2151), rapamycin $(0.5 \mu \mathrm{M}$, Selleckchem, S1039) were employed to inhibit PI3K, AKT, and mTOR, respectively.

\section{Backscattered and resin-casted scanning electron microscopy}

Extracted and cleaned molars were fixed and cut longitudinally. Acid etching was performed with $37 \%$ phosphoric acid for $5 \mathrm{~s}$ and followed by washing with $5.25 \%$ sodium hypochlorite for $5 \mathrm{~min}$ on the dentin surface as described before ${ }^{44}$. The specimens were dehydrated in a graded ethanol series $(30 \%, 50 \%, 70 \%, 85 \%$, and $100 \%$ for $15 \mathrm{~min}$ ) and washed with hexamethyldisilizane. The samples were air dried overnight and then coated with carbon. A Hitachi SU70 scanning electron microscope (Hitachi Instruments, Schaumburg, IL, USA) was used to perform the analyses.

\section{Histology}

Mouse mandibles were excised, fixed with $10 \%$ naturalbuffered formalin (VWR International, West Chester, PA, USA), and decalcified in 10\% EDTA (ethylenediaminetetraacetic acid, Thermo Fisher Scientific) for 2 weeks at $4{ }^{\circ} \mathrm{C}$. Paraffin-embedded samples were sectioned and stained with hematoxylin and eosin.

\section{DPSC isolation, culture, and differentiation}

The incisors were isolated from the mandibles that were dissected from 6-week-old IFT80 $0^{f / f}$ mice. Whole dental pulp was gently collected from the interior of the incisor and exposed to enzymatic digestion with collagenase type I $(3 \mathrm{mg} / \mathrm{mL})$ and dispase $(4 \mathrm{mg} / \mathrm{mL})$ for $1 \mathrm{~h}$ at $37^{\circ} \mathrm{C}$ with shaking. The digested tissues were homogenized by repetitive pipetting and the released cells were centrifuged at $200 \times g$ for $10 \mathrm{~min}$. The cells were cultured in $\alpha$-modified Eagle's medium ( $\alpha$-MEM, Life Technologies) containing 10\% fetal bovine serum (FBS, Life Technologies), $2 \mathrm{mM}$ L-glutamine (Life Technologies), $100 \mathrm{U} / \mathrm{mL}$ penicillin, and $100 \mu \mathrm{g} / \mathrm{mL}$ streptomycin (Life Technologies). DPSCs could adhere to the plastic dish for $24 \mathrm{~h}$ and then the medium was changed to remove floating debris. Culture medium was replaced every 3 days until the cells reach $80 \%$ confluence. Then, the cells were detached by $0.25 \%$ Trypsin-EDTA (Life Technologies) and subcultivated at a ratio of $1: 2^{45,46}$.

DPSCs from IFT80 $0^{f f}$ mice were infected with adenovirus that overexpress either Cre (Ad-CMV-Cre, \#1405, Vector Biolabs) or GFP (Ad-GFP, \#1060, Vector Biolabs). Ad-CMV-Cre infection causes IFT80 deletion in IFT8f fff DPSCs, which were then marked as IFT80 ${ }^{d / d}$. Ad-GFP were used as an infection control and Ad-GFP-treated IFT80 $0^{f f f}$ DPSCs were still marked as IFT8 $80^{f / f}$.

\section{Western blot}

Cells were harvested and homogenized with radioimmunoprecipitation assay buffer. Proteins were denatured in sodium dodecyl sulfate (SDS) buffer and separated with SDS-polyacrylamide gel electrophoresis 
gels. Proteins were transferred to polyvinylidene difluoride membranes and then blocked with 5\% skimmed milk (OXOID). Membranes were incubated with primary antibody overnight at $4{ }^{\circ} \mathrm{C}$, and then incubated with horseradish peroxidase (HRP)-conjugated goat anti-rabbit immunoglobulin G (IgG) antibody (1:10,000, Novex, Carlsbad, CA, USA) at room temperature for $1 \mathrm{~h}$. Visualization was performed with WesternBright ECL HRP (Bio-Rad). $\beta$-Actin (1:500, Santa Cruz) was used as the internal control.

The same procedure was used to determine the IFT80 (1:400, PAB15842, Abnova), AKT (1:300, sc-8312, Santa Cruz), and p-AKT (1:300, sc-7985-r, Santa Cruz).

\section{Quantitative PCR}

Total RNA was extracted from cultured DPSCs with Trizol reagent (Invitrogen, Carlsbad, CA, USA) and then synthesized to complementary DNA (cDNA) with total RNA by RNA to cDNA EcoDry Premix Kit (Clontech, Palo Alto, CA, USA). qPCR was performed with ABI PRISM 7500 Real-Time PCR Machine (Invitrogen, Carlsbad, CA, USA) and SYBR Green PCR Master Mix (Invitrogen). Sequence and product length for each primer pair were listed in Supplementary Table S1. Gene expression was normalized to the housekeeping gene GAPDH and calculated according to the $2^{\text {-ddCT }}$ method $^{47}$. All reactions were run in triplicate.

\section{Immunocytochemistry and immunofluorescence}

Deparaffinized sections or fixed cells were permeabilized, blocked, and incubated with primary anti-Ki67 antibody (1:50 for slides, 1:200 for cells, ab16667) overnight at $4{ }^{\circ} \mathrm{C}$. The slides were washed and stained with Alexa Fluor 568-conjugated anti-rabbit IgG (1:1000, Invitrogen) antibody or for $1 \mathrm{~h}$ at room temperature. DAPI (6-diamidino-2-phenylindole, Sigma) was used as a counterstain for nuclei .

The same staining procedure was used for acetylated $\alpha$ tubulin (1:500, T6793, Sigma). For primary cilia staining of DPSCs, the cells were serum starved for $48 \mathrm{~h}$ to induce ciliogenesis.

\section{MTS assay}

The MTS assay was performed using the CellTiter $96^{\circ}$ AQueous One Solution Cell Proliferation Assay Kit (Promega).

\section{Reporter assay}

To test Gli promoter activity, reporter assay was performed with Gli-responsive luciferase reporter construct $\left(8 \times\right.$ Gli-Luc) (gift from Dr. Fernandez-Zapico $\left.{ }^{48}\right)$. DPSCs $\left(1 \times 10^{6}\right)$ were transfected with $3 \mu \mathrm{g} 8 \times$ Gli-Luc and $0.6 \mu \mathrm{g}$ pRL-TK Renilla luciferase (Promega, internal control) with Fugene HD transfection reagent. Cells were induced with OS medium for 3 days and then stimulated with $1 \mu \mathrm{g} / \mathrm{mL}$ of recombinant mouse Shh N-terminus (R\&D Systems, Minneapolis, MN, USA) for $8 \mathrm{~h}$. Cells were harvested and colored by the Dual-luciferase Assay Kit (Promega, Madison, WI, USA) and the relative luciferase activity was measured with the Veritas microplate luminometer (Turner Biosystem, Sunnyvale, CA, USA). The experiment was conducted in triplicate.

\section{Statistical analysis}

All data are presented as mean $\pm \operatorname{SEM}(n=3$ or more as indicated in figure legends). Comparisons between two groups were performed by Student's $t$ test and comparisons among grouped samples were analyzed by two-way analysis of variance followed by Tukey's multiple comparison. $P<0.05$ was considered to be statistically significant. The program GraphPad Prism (GraphPad Software Inc., San Diego, CA, USA) was used for these analyses.

\section{Acknowledgements}

We thank Dr. Aimee B. Stablewski and Gene Targeting and Transgenic Shared Resource at Roswell Park Cancer Institute (supported by the National Cancer Institute (NCI) grant P30CA016056) for technical assistance with the ES cell injections for generating IFT80-floxed mice. We also thank Dr. Songtao Shi for the critical reading and suggestion in the manuscript.

\section{Author details}

'Department of Oral Biology, School of Dental Medicine University of Buffalo, State University of New York, Buffalo, NY, USA. ${ }^{2}$ Department of Orthopedic Surgery, Johns Hopkins University School of Medicine, Baltimore, MD, USA. ${ }^{3}$ Department of Anatomy and Cell Biology, School of Dental Medicine, University of Pennsylvania, Philadelphia, PA, USA

Conflict of interest

The authors declare that they have no conflict of interest.

\section{Publisher's note}

Springer Nature remains neutral with regard to jurisdictional claims in published maps and institutional affiliations.

Supplementary Information accompanies this paper at (https://doi.org/ 10.1038/s41419-018-0951-9).

Received: 23 April 2018 Accepted: 1 August 2018

Published online: 25 January 2019

\footnotetext{
References

1. Li, J., Parada, C. \& Chai, Y. Cellular and molecular mechanisms of tooth root development. Development 144, 374-384 (2017).

2. Sharpe, P. T. Dental mesenchymal stem cells. Development 143, 2273-2280 (2016).

3. Goetz, S. C. \& Anderson, K. V. The primary cilium: a signalling centre during vertebrate development. Nat. Rev. Genet. 11, 331-344 (2010).

4. Satir, P., Pedersen, L. B. \& Christensen, S. T. The primary cilium at a glance. J. Cell. Sci. 123(Part 4), 499-503 (2010).

5. Huber, C. \& Cormier-Daire, V. Ciliary disorder of the skeleton. Am. J. Med. Genet. C 160, 165-174 (2012).

6. Haycraft, C. J. \& Serra, R. Cilia involvement in patterning and maintenance of the skeleton. Curr. Top. Dev. Biol. 85, 303-332 (2008).
} 
7. Nakatomi, M. et al. Evc regulates a symmetrical response to Shh signaling in molar development. J. Dent. Res. 92, 222-228 (2013).

8. Thivichon-Prince, B. et al. Primary cilia of odontoblasts: possible role in molar morphogenesis. J. Dent. Res. 88, 910-915 (2009).

9. Liu, B., Chen, S., Cheng, D., Jing, W. \& Helms, J. A. Primary cilia integrate hedgehog and Wnt signaling during tooth development. J. Dent. Res. 93 475-482 (2014).

10. Kolpakova-Hart, E., Jinnin, M., Hou, B., Fukai, N. \& Olsen, B. R. Kinesin-2 controls development and patterning of the vertebrate skeleton by Hedgehog- and Gli3-dependent mechanisms. Dev. Biol. 309, 273-284 (2007).

11. Ruiz-Perez, V. L. et al. Evc is a positive mediator of Ihh-regulated bone growth that localises at the base of chondrocyte cilia. Development 134, 2903-2912 (2007).

12. Ohazama, A. et al. Primary cilia regulate Shh activity in the control of molar tooth number. Development 136, 897-903 (2009).

13. Zhang, Q. et al. Loss of the $\mathrm{Tg} 737$ protein results in skeletal patterning defects. Dev. Dyn. 227, 78-90 (2003).

14. Yang, S. \& Wang, C. The intraflagellar transport protein IFT80 is required for cilia formation and osteogenesis. Bone 51, 407-417 (2012).

15. Yuan, X. et al. Ciliary IFT80 balances canonical versus non-canonical hedgehog signalling for osteoblast differentiation. Nat. Commun. 7, 11024 (2016).

16. Hatch, N. E. FGF signaling in craniofacial biological control and pathological craniofacial development. Crit. Rev. Eukaryot. Gene Expr. 20, 295-311 (2010).

17. Russo, L. G., Maharajan, P. \& Maharajan, V. Basic fibroblast growth factor (FGF-2) in mouse tooth morphogenesis. Growth Factors 15, 125-133 (1998).

18. Sagomonyants, K., Kalajzic, I., Maye, P. \& Mina, M. Enhanced dentinogenesis of pulp progenitors by early exposure to FGF2. J. Dent. Res. 94, 1582-1590 (2015).

19. Kim, S. G. et al. Effects of growth factors on dental stem/progenitor cells. Dent Clin. N. Am. 56, 563-575 (2012)

20. Kim, J. et al. Treatment of FGF-2 on stem cells from inflamed dental pulp tissue from human deciduous teeth. Oral Dis. 20, 191-204 (2014).

21. Osathanon, T., Nowwarote, N. \& Pavasant, P. Basic fibroblast growth factor inhibits mineralization but induces neuronal differentiation by human denta pulp stem cells through a FGFR and PLCY signaling pathway. J. Cell. Biochem. 112, 1807-1816 (2011).

22. Sagomonyants, K, Kalajzic, I., Maye, P. \& Mina, M. FGF signaling prevents the terminal differentiation of odontoblasts. J. Dent. Res. 96, 663-670 (2017).

23. Mizoguchi, T. et al. Osterix marks distinct waves of primitive and definitive stromal progenitors during bone marrow development. Dev. Cell 29, 340-349 (2014).

24. Rakian, A. et al. Bone morphogenetic protein-2 gene controls tooth root development in coordination with formation of the periodontium. Int. J. Oral Sci. 5, 75-84 (2013).

25. Wang, Y., Cox, M. K., Coricor, G., MacDougall, M. \& Serra, R. Inactivation of Tgfbr2 in Osterix-Cre expressing dental mesenchyme disrupts molar root formation. Dev. Biol. 382, 27-37 (2013).

26. Su, N., Jin, M. \& Chen, L. Role of FGF/FGFR signaling in skeletal development and homeostasis: learning from mouse models. Bone Res. 2, 14003 (2014).

27. Magloire, H., Couble, M. L., Romeas, A. \& Bleicher, F. Odontoblast primary cilia: facts and hypotheses. Cell Biol. Int. 28, 93-99 (2004).

28. Yuan, X. \& Yang, S. Primary cilia and intraflagellar transport proteins in bone and cartilage. J. Dent. Res. 95, 1341-1349 (2016).
29. Hampl, M. et al. Role of primary cilia in odontogenesis. J. Dent. Res. 96 965-974 (2017).

30. Li, G. et al. Essential role of IFT140 in promoting dentinogenesis. J. Dent. Res. 97, 423-431 (2017)

31. Hisamoto, M. et al. Developmental changes in primary cilia in the mouse tooth germ and oral cavity. Biomed. Res. 37, 207-214 (2016).

32. Christensen, S. T., Clement, C. A., Satir, P. \& Pedersen, L. B. Primary cilia and coordination of receptor tyrosine kinase (RTK) signalling. J. Pathol. 226, 172-184 (2012)

33. Huangfu, D. et al. Hedgehog signalling in the mouse requires intraflagellar transport proteins. Nature $\mathbf{4 2 6}, 83-87$ (2003).

34. Robbins, D. J., Fei, D. L. \& Riobo, N. A. The hedgehog signal transduction network. Sci. Signal. 5, re6 (2012).

35. Yuan, X. \& Yang, S. Cilia/lft protein and motor -related bone diseases and mouse models. Front. Biosci. 20, 515-555 (2015)

36. Yuan, X. \& Yang, S. Deletion of IFT80 impairs epiphyseal and articular cartilage formation due to disruption of chondrocyte differentiation. PLOS ONE 10, e0130618 (2015).

37. Wang, C. D., Yuan, X. \& Yang, S. Y. IFT80 is essential for chondrocyte differentiation by regulating Hedgehog and Wnt signaling pathways. Exp. Cell Res. 319, 623-632 (2013).

38. Noda, K., Kitami, M., Kitami, K., Kaku, M. \& Komatsu, Y. Canonical and noncanonical intraflagellar transport regulates craniofacial skeletal development. Proc. Natl. Acad. Sci. USA 113, E2589-E2597 (2016).

39. Grisanti, L., Revenkova, E., Gordon, R. E. \& Iomini, C. Primary cilia maintain corneal epithelial homeostasis by regulation of the Notch signaling pathway. Development. dev-132704 (2016).

40. Yeh, C. et al. IGF-1 activates a cilium-localized noncanonical Gbetagamma signaling pathway that regulates cell-cycle progression. Dev. Cell 26, 358-368 (2013).

41. Dalbay, M. T., Thorpe, S. D., Connelly, J. T., Chapple, J. P. \& Knight, M. M. Adipogenic differentiation of hMSCS is mediated by recruitment of IGF-1r onto the primary cilium associated with cilia elongation. Stem Cells $\mathbf{3 3}$ 1952-1961 (2015).

42. Neugebauer, J. M., Amack, J. D., Peterson, A. G., Bisgrove, B. W. \& Yost, H. J. FGF signalling during embryo development regulates cilia length in diverse epithelia. Nature 458, 651-654 (2009).

43. Sagomonyants, K. \& Mina, M. Stage-specific effects of fibroblast growth factor 2 on the differentiation of dental pulp cells. Cells Tissues Organs 199, 311-328 (2014).

44. Zhu, Q. et al. Proteolytic processing of dentin sialophosphoprotein (DSPP) is essential to dentinogenesis. J. Biol. Chem. 287, 30426-30435 (2012).

45. Akmal, M. et al. Isolation and characterization of dental pulp stem cells from murine incisors. J. Biol. Sci. 14, 571-574 (2014).

46. Ellis, K. M., O'Carroll, D. C., Lewis, M. D., Rychkov, G. Y. \& Koblar, S. A. Neurogenic potential of dental pulp stem cells isolated from murine incisors. Stem Cell Res. Ther. 5, 30 (2014)

47. Livak, K. J. \& Schmittgen, T. D. Analysis of relative gene expression data using real-time quantitative PCR and the 2(-Delta Delta C(T)) Method. Methods 25 402-408 (2001)

48. Kurita, S. et al. GLI3-dependent repression of DR4 mediates hedgehog antagonism of TRAlL-induced apoptosis. Oncogene 29, 4848-4858 (2010). 